\title{
Sistema de Gestión de Calidad para el mejoramiento de los procesos de producción de Babaco
}

\section{Quality Management System for the improvement of Babaco's production processes}

Juan Gabriel Chipantiza-Masabanda juan.chipantiza@espoch.edu.ec

Escuela Superior Politécnica de Chimborazo, Riobamba

Ecuador

https://orcid.org/0000-0002-7005-9134

Amanda Elizabeth Bonilla-Bonilla

amanda.bonilla@espoch.edu.ec

Escuela Superior Politécnica de Chimborazo, Riobamba

Ecuador

https://orcid.org/0000-0002-9429-1245

Byron Stalin Rojas-Oviedo

stalinrojas96@espoch.edu.ec

Escuela Superior Politécnica de Chimborazo, Riobamba

Ecuador

https://orcid.org/0000-0003-2415-6205

Recibido: 15 de agosto de 2021

Aprobado: 15 de noviembre de 2021 


\title{
RESUMEN
}

El presente trabajo de investigación tiene como objetivo proponer el sistema de gestión de calidad para el mejoramiento de los procesos de producción del babaco en la Asociación de Productores Autónomos Rio Chico el Rosal. El enfoque de la investigación es cuantitativo, el estudio fue de campo, exploratoria y descriptiva. La población y la muestra estuvo conformado 40 socios y trabajadores de la asociación de productores. De acuerdo al diagnóstico de los procesos de producción por medio de los diagramas de procesos de producción existen demoras, inspecciones, transporte, operaciones, innecesarias, largos tiempos de producción, mismos que son causados por la inexistencia de procedimientos que estandaricen las actividades a realizar. Se identificó los lineamientos de calidad del producto valorado fundamentalmente por su uniformidad, madurez y ausencia de defectos, el color, la viscosidad y el rendimiento industrial como materia prima del producto terminado y se diseñó el proceso de producción del babaco.

Descriptores: Gestión; calidad del entorno; proceso de producción. (Palabras tomadas del Tesauro UNESCO).

\begin{abstract}
The objective of this research work is to propose the quality management system for the improvement of the babaco production processes in the Association of Autonomous Producers Rio Chico el Rosal. The research approach is quantitative, the study was field, exploratory and descriptive. The population and the sample consisted of 40 members and workers of the producers' association. According to the diagnosis of the production processes through the production process diagrams, there are delays, inspections, transportation, unnecessary operations, long production times, which are caused by the lack of procedures that standardize the activities to be carried out. The quality guidelines of the product valued fundamentally for its uniformity, maturity and absence of defects, color, viscosity and industrial performance as raw material of the finished product were identified and the babaco production process was designed.
\end{abstract}

Descriptors: Management; quality of the environment; production process. (Words taken from UNESCO Thesaurus). 


\section{INTRODUCCIÓN}

La fortaleza de una organización se mide en su poder de perfeccionar y aumentar sus capacidades, actitudes, conocimientos, experiencias y ambición de crecimiento. Las organizaciones fuertes son las que llevan a la comunidad a buen vivir y buscan el bienestar común. El fortalecimiento de las capacidades de la asociación de Productores Autónomos Río Chico El Rosal es el proceso para incrementar la visión del futuro, capacidad de gestión, sostenibilidad, capacidad administrativa y actitud asociativa y de compromiso. Para ello se desarrollará un manual de sistema de gestión de la calidad de babaco, que les permita eliminar debilidades y robustecer a la organización.

La asociación de estos productores de Río Chico El Rosal fue fundada en el año de 1987 cuyos fines es la producción agropecuaria, actualmente se dedican a la producción de babaco bajo invernadero, lo cual les ha permitido sustentarse a través de los años. En esta actividad se han identificado ineficiencia en los procesos productivos, causando desorientación en su visión, misión y objetivos, también sumando a esto problemas administrativos y financieros.

Es importante destacar, que el progreso de las empresas dedicadas a la producción del babaco en el mundo hace que la calidad se muestre como piedra angular de las estrategias de las empresas, aportando metodologías, herramientas, experiencias y buenas prácticas que dan respuesta a los retos que el nuevo escenario económico plantea a las organizaciones (Montilla \& Herrera, 2006).

El sistema de gestión de la calidad, es aplicable a todo tipo de empresas (pequeñas, medianas, grandes), ya que permite identificar y establecer sistemas, estandarizar los procesos productivos con la finalidad desde llegar a cubrir las necesidades de los clientes y por ende posicionarse en el mercado (González, 2011).

El sistema de gestión de la calidad en la última década ha ayudado a los procesos de producción a disminuir la calidad de los productos no conformes, a evitar reproceso, desperdicios de materia prima, tiempos elevados de producción, tiempos muertos de producción, entre otros (Casañas, 2015). 
En cuanto a la contextualización meso, se tiene que la provincia de Tungurahua cuenta con un alto índice de empresas, asociaciones de agricultores dedicadas a la producción y comercialización del babaco, las mismas que tienen una producción no sofisticada en las que existen algunos inconvenientes como la deficiencia en el manejo de la documentación y registros con los que se debe conocer y controlar apropiadamente los procesos de producción; es decir, no existe un control de calidad durante los procesos, tampoco existe la implementación de un Sistema de gestión de calidad enfocado en la normas ISO 9001: 2015 (Guaipatin \& Schwartz, 2014). Por esta razón se ha puesto un énfasis por parte de las empresas (pequeñas, medianas, grandes), asociaciones en temas de calidad, el mejoramiento continuo y la implementación del sistema de Gestión de Calidad, con el objetivo de lograr la satisfacción del cliente (Pérez, 2014, p. 3).

Por medio de la implementación de un sistema de gestión de la calidad, las empresas y asociaciones dedicadas a la producción del babaco, han logrado mejorar la calidad del producto y posicionar su marca en el mercado de la provincia de Tungurahua, logrando mejorar la rentabilidad de los mismos. Según el Instituto de Investigaciones Agropecuarias (2012).

En lo respecta a la contextualización micro, la Asociación de Productores Autónomos de Rio Chico el Rosal, ubicada en el cantón Patate es una asociación dedicada a la producción y comercialización del babaco, fue creada en el 2010 con trayectoria de 7 años en el mercado local, con el pasar del tiempo la asociación incremento su demanda de producción, es por ello que el gerente de la asociación ha dirigido sus esfuerzos para el cumplimiento de los requisitos establecidos en la norma y la satisfacción del cliente encaminándose hacia la gestión de la calidad.

La asociación no posee un sistema de gestión de calidad, lo que hace que no garantice a sus clientes un producto optimo y competitivo en el mercado, además carece de procesos no estandarizados, es decir los trabajos que se realizan son de forma empírica, cada trabajador ejecuta sus actividades en base a la experiencia adquirida, creando fragilidad latente a la hora de desarrollar los procesos de producción. Para 
esto es indispensable que las actividades desarrolladas estén correctamente documentadas (Mateo, 2010).

\section{Sistema de Gestión de la Calidad}

El sistema de gestión se encarga de dirigir, controlar una organización con lo referente a la calidad, de tal forma que el SGC es el conjunto de reglas y la forma de trabajar a la que se rige una empresa. Según (ISO 9000; 2005, 2005), además la. Este sistema comprende la estructura organizacional, conjuntamente con la planificación, los procesos, los recursos, los documentos que necesitamos para alcanzar los objetivos de la organización para proveer mejoramiento de productos y servicios y para cumplir los requerimientos de nuestros clientes.

Según Mateo (2010) afirma que un sistema de gestión de la calidad de una organización está formado por un conjunto de elementos tales como: las estrategias, los objetivos, las políticas, los métodos, los recursos y las estructuras, la tecnología, las instrucciones del trabajo, los procedimientos, los procesos, a través del cual la dirección de la organización planifica, ejecuta y controla, cada una de sus actividades, con la finalidad de lograr sus objetivos planteados.

En el sistema de gestión de la calidad se identifican los diferentes procesos del que está conformado la empresa, en la cual se establece la secuencia de interacción de acuerdo a Gutiérrez (2010, pág. 63).

\section{Gestión de la Calidad}

Para Camisón, Cruz, \& González (2014), la gestión de la calidad es una colección de varios métodos, utilizables puntualmente y aisladamente para el control de la calidad de un producto/servicio, durante sus procesos productivos. Esto implica que su personal (profesorado y personal de administración y servicios), también puede tomar decisiones. Los principios de la Gestión de Calidad son adoptados por las organizaciones para realizar la calidad de sus productos y servicios y de esta manera aumentar su eficiencia. 
Para Cuatrecasa (2012) La gestión de la calidad "en los últimos años ha ido evolucionando a un ritmo vertiginoso, encaminado en gestionar todos los procesos de una empresa, basándose o partiéndose de la calidad, que permitirá conseguir ventajas competitivas y la satisfacción de los clientes" (p. 578)

Los autores Camisón \& Cruz (2010) señalan que la gestión de calidad es la forma de gestionar, para que se adapte al entorno competitivo actual, el hecho de que en una empresa concreta sea una moda pasajera o se convierta una manera habitual de funcionar, depende de numerosos factores. Se suele citar como el más importante la implicación de la dirección; estamos d acuerdo. Pero hay otro factor de importancia capital: las personas y su capacidad de afectar la organización a través de sus habilidades y conocimientos.

\section{Norma ISO 9001: 2015}

Según ISO 9001: 2015 (2015) Esta norma internacional promueve la adopción de un enfoque basado en procesos cuando se desarrolla, implementa y mejora la eficacia de un sistema de gestión de la calidad, para aumentar la satisfacción del cliente mediante el cumplimiento de sus requisitos.

Para Auquillas (2016) La norma ISO 9001: 2015 es actualmente el estándar internacional certificable para los sistemas de gestión de la calidad. La nueva versión aprobada en septiembre del 2015 presenta cambios considerables respecto a la versión anterior (ISO 9001:2008). Básicamente se puede indicar que ahora se cuenta con 10 capítulos a diferencia de la versión anterior que eran únicamente 8.

\section{METODOLOGÍA}

La presente investigación se basó en un enfoque cualicuantitativo (cuantitativo y cualitativo). El Enfoque cuantitativo según Hernández, Fernández, \& Baptista (2013, pág. 4) es el uso de la recolección de datos para probar hipótesis, con base en la medición numérica y el análisis estadístico, para establecer patrones de comportamiento y probar teorías. Es decir, porque se determinará la realidad del 
proceso de producción de la Asociación de Productores Autónomos Rio Chico El Rosal. Estos datos facilitaran el análisis del lugar donde se ocasionan los problemas, sus causas por las que se generan los defectos en la producción del babaco.

El Enfoque cualitativo según Hernández, Fernández, \& Baptista (2013, p. 7) define como utiliza la recolección de datos sin medición numérica para descubrir o afinar preguntas de investigación en el proceso de interpretación. Es decir, porque la investigación se realizó en el lugar de los hechos, donde participan todos los integrantes que conforman la Asociación de Productores Autónomos Rio Chico El Rosal.

\section{Modalidad básica de la investigación}

La Investigación bibliográfica de acuerdo a Hernández, Fernández, \& Baptista (2013, p. 15) afirman que este tipo de investigación facilita una mayor penetración y comprensión del problema investigado, de tal forma que se recurrió a fuentes bibliográficas primarias y secundarias como textos, revistas, artículos científicos, estudios realizados, referentes al tema de investigación.

La Investigación de campo para los autores Hernández, Fernández, \& Baptista (2013, p. 16) es un proceso que utiliza el método científico, con la finalidad de diagnosticar el problema. Es decir, para desarrollar un sistema de gestión de la calidad fue necesario acudir al lugar de los hechos, es decir a la asociación de Productores Autónomos Rio Chico El Rosal. Con el fin de obtener información detalla del problema.

\section{Nivel o tipo de investigación}

Investigación exploratoria: Permitió conocer el tema de investigación y el problema de forma acertada. Además, proporcionará alternativas para el desarrollo del presente trabajo.

Investigación descriptiva transversal: Midió y recogió la información relevante del Sistema de Gestión de la Calidad y a los procesos de producción del babaco además se buscará a través de las encuestas la percepción del problema. 


\section{Población y muestra}

Para la elaboración del presente proyecto se tomó en cuenta a todos los socios y a trabajadores de la asociación de Productores Autónomos Rio Chico El Rosal de la parroquia el Triunfo cantón Patate.

\section{RESULTADOS}

\section{Análisis de la encuesta realizada a los integrantes de la Asociación}

Pregunta 1: ¿La asociación cumple con la entrega de productos en el tiempo acordado?

\section{Tabla 1.}

Cumple la entrega de pedidos a tiempo.

\begin{tabular}{|l|l|l|}
\hline Alternativa & Frecuencia & Porcentaje \\
\hline Siempre & 0 & $0,00 \%$ \\
\hline Casi siempre & 33 & $82,50 \%$ \\
\hline Algunas veces & 3 & $7,50 \%$ \\
\hline Nunca & 4 & $10,00 \%$ \\
\hline Total & 40 & $100,00 \%$ \\
\hline
\end{tabular}

Fuente: Encuesta aplicada a los socios y trabajadores. Chipantiza (2017).

\section{Análisis e interpretación}

Según la información de la tabla 1, del total de personas encuestadas el $82 \%$ afirmaron que la asociación algunas veces cumple a tiempo con los pedidos, el 10\% indicaron que nunca la asociación cumple a tiempo con los pedidos, y el $8 \%$ afirmaron que algunas veces la asociación si cumple con la entrega de pedidos a tiempo. 
De acuerdo a la información obtenida de la figura anterior, la mayoría de las personas dieron a conocer que la asociación la mayoría de veces si cumplen con la entregan de pedidos a tiempo.

Pregunta 2: ¿Qué percepción tiene Usted con respecto a la gestión de quejas, reclamos y devoluciones, por parte del personal de ventas de la Asociación?

\section{Tabla 2.}

Percepción de quejas, reclamos y devoluciones.

\begin{tabular}{|l|l|l|}
\hline Alternativa & Frecuencia & Porcentaje \\
\hline $\begin{array}{l}\text { Cumple en parte las } \\
\text { expectativas }\end{array}$ & 16 & $40,00 \%$ \\
\hline $\begin{array}{l}\text { No cumple con las } \\
\text { expectativas }\end{array}$ & 24 & $60,00 \%$ \\
\hline $\begin{array}{l}\text { Supera algunas } \\
\text { expectativas }\end{array}$ & 0 & $0,00 \%$ \\
\hline Total & 40 & $100,00 \%$ \\
\hline
\end{tabular}

Fuente: Encuesta aplicada a los socios y trabajadores. Chipantiza (2017).

\section{Análisis e interpretación}

Según el resultado obtenido en la tabla 2 , el $60 \%$ señalaron que no cumplen con las expectativas la asociación con respecto a los clientes; mientras que el $40 \%$ afirmaron que la asociación si cumple en parte con las expectativas de los clientes.

Con respecto a la figura anterior, se puede describir que la mayoría de los trabajadores encuestados tienen una perspectiva que la asociación en si no cumple con las expectativas de los clientes en cuanto al producto, debido a que ellos al momento de realizar el contrato se finan otras características, y al momento de entregar el producto son otras, es por esto que existe muchos reclamos por parte de los clientes. 
Pregunta 3: ¿La Asociación de productores Autónomos Rio Chico el Rosal, comunica a los clientes los parámetros de calidad del producto?

Tabla 3.

La Asociación comunica a los clientes de calidad del producto.

\begin{tabular}{|l|l|l|}
\hline Alternativa & Frecuencia & Porcentaje \\
\hline $\mathrm{Si}$ & 25 & $62,50 \%$ \\
\hline No & 15 & $37,50 \%$ \\
\hline Total & 40 & $100,00 \%$ \\
\hline
\end{tabular}

Fuente: Encuesta aplicada a los socios y trabajadores. Chipantiza (2017).

\section{Análisis e interpretación}

De acuerdo a la tabla 3 , se puede observar que un $62 \%$ señalaron la Asociación de Productores Autónomos Rio Chico el Rosal, si comunica a los clientes sobre la calidad del producto, mientras que el $38 \%$ afirmaron que la asociación no comunica a los clientes sobre la calidad del producto.

La mayoría de los encuestados dieron a conocer que la asociación de Productores Autónomos Rio Chico el Rosal, antes de ofertar si da a conocer los respectivos clientes las características como, por ejemplo, el color, el tamaño, el peso, la forma, por ende, la calidad del producto, muchas veces el personal de venta cuenta con un el producto para que el cliente pueda observar. 
Pregunta 4: ¿Considera usted que el personal que labora en la asociación tiene el nivel de estudio acorde a su puesto de trabajo?

\section{Tabla 4.}

Personal de trabajo cuenta con el nivel de estudio apropiado.

\begin{tabular}{|l|l|l|}
\hline Alternativa & Frecuencia & Porcentaje \\
\hline $\mathrm{Si}$ & 27 & $67,50 \%$ \\
\hline No & 13 & $32,50 \%$ \\
\hline Total & 40 & $100,00 \%$ \\
\hline
\end{tabular}

Fuente: Encuesta aplicada a los socios y trabajadores. Chipantiza (2017).

\section{Análisis e interpretación}

El resultado obtenido en la tabla 4, muestra que el 67\% dijeron que la Asociación si cuenta con el personal con un estudio acorde a su puesto de trabajo, mientras que el $33 \%$ afirmó que el personal que labora en la asociación no cuenta con el nivel de estudio acorde al área de trabajo.

La Asociación de productores Autónomos Rio Chico el Rosal si cuenta con el personal idóneo para el desarrollo de las diferentes actividades, para la producción del babaco, de tal forma la asociación en la parte administrativa cuenta con un personal de estudio de tercer nivel; mientras que en la parte productiva la mayoría del personal se encuentra cursando un nivel de tecnologado y una minoría del personal cuenta con un nivel de bachillerato. 
Pregunta 5: ¿Conoce Usted si el personal se encuentra capacitado, para la producción del babaco en la asociación?

Tabla 5.

Porcentaje de personal capacitado.

\begin{tabular}{|l|l|l|}
\hline Alternativa & Frecuencia & Porcentaje \\
\hline $\mathrm{Si}$ & 9 & $22,50 \%$ \\
\hline No & 31 & $77,50 \%$ \\
\hline Total & 40 & $100,00 \%$ \\
\hline
\end{tabular}

Fuente: Encuesta aplicada a los socios y trabajadores. Chipantiza (2017).

\section{Análisis e interpretación}

De acuerdo a los resultados obtenidos en la tabla 5 , el $78 \%$ señalaron que no conocen el número del personal que haya sido capacitado para que labore en la asociación; mientras que el $22 \%$ afirmaron que si conocen el número del personal capacitado que labora en la asociación.

De acuerdo a la figura anterior señala que la mayoría no conoce a ciencia cierta la cantidad del personal capacitado, ya que manifiestan que la asociación casi no realiza capacitaciones, en el mejor de los casos la asociación ha realizado charlas de acuerdo a las necesidades presentadas durante la ejecución de las actividades. La persona encargada de la charla es el jefe de producción. Razón por la cual las falencias continúan.

Pregunta 6: ¿Considera que los recursos asignados para la producción del babaco en la asociación de productores Autónomos Rio Chico el Rosal, son los adecuados? 


\section{Tabla 6.}

Los recursos asignados para la producción del babaco son los adecuado.

\begin{tabular}{|l|l|l|}
\hline Alternativa & Frecuencia & Porcentaje \\
\hline $\mathrm{Si}$ & 17 & $42,50 \%$ \\
\hline No & 23 & $57,50 \%$ \\
\hline Total & 40 & $100,00 \%$ \\
\hline
\end{tabular}

Fuente: Encuesta aplicada a los socios y trabajadores. Chipantiza (2017).

\section{Análisis e interpretación}

Según los datos obtenidos en la tabla 6 ; el $58 \%$ señalaron que no son adecuados los recursos asignados para la producción del babaco; mientras que el $42 \%$ de los trabajadores afirmaron que si son adecuados los recursos asignados para la producción del babaco.

La mayoría de los trabajadores consideran que los recursos asignados para la producción del babaco no son adecuados, ya que en la mayoría de los casos la asociación solo entrega recursos técnicos, pero recursos financieros y humanos casi siempre no es el apropiado, ya que existe sobrecarga de trabajo y materiales e insumos de mala calidad.

Pregunta 7: ¿Conoce la cantidad de materia prima necesaria, que requiere la Asociación de productores Autónomos Rio Chico el Rosal, para un lote de producción?

Tabla 7.

Cantidad de materia prima requerida.

\begin{tabular}{|l|l|l|}
\hline Alternativa & Frecuencia & Porcentaje \\
\hline $\mathrm{Si}$ & 23 & $57,50 \%$ \\
\hline No & 17 & $42,50 \%$ \\
\hline Total & 40 & $100,00 \%$ \\
\hline
\end{tabular}

Fuente: Encuesta aplicada a los socios y trabajadores. Chipantiza (2017) 


\section{Análisis e interpretación}

En la tabla 7, se observa que el $57 \%$ de los encuestados señalaron que si conocen la cantidad de materia prima requerida para la producción de un lote de babaco; mientras que el $43 \%$ afirmaron que no conocen la cantidad de materia prima requerida necesaria para el área de producción. De acuerdo a la mayoría de los encuestados si conocen la cantidad exacta de la materia prima para el área de producción del babaco, pero afirman que la asociación no siempre cumple con la disponibilidad de dicha cantidad, razón por la cual, en varias ocasiones, se ha tenido que sembrar después del día establecido.

\section{Verificación de Hipótesis}

Para la verificación del chi cuadrado $\left(x^{2}\right)$ se utilizó formulas estadísticas y lograr demostrar si existe una relación entre las dos variables (variable independiente; variable dependiente)

HO: El Sistema de Gestión de Calidad no mejorará los procesos de producción del babaco de la Asociación de productores Autónomos Rio Chico el Rosal.

H1: El Sistema de Gestión de Calidad mejorará los procesos de producción del babaco de la Asociación de productores Autónomos Rio Chico el Rosal.

\section{Tabla 8.}

Tabla cruzada del chi cuadrado.

\begin{tabular}{|l|l|l|l|l|l|l|l|}
\hline & Preg. 1 & Preg. 2 & Preg. 3 & Preg. 4 & Preg. 5 & Preg. 6 & Preg. 7 \\
\hline Preg. 1 & 0 & 7,944 & 5,996 & 8,872 & 18,182 & 24,259 & 16,233 \\
\hline Preg. 2 & 7,944 & 0 & 11,11 & 5,511 & 3,81 & 6,273 & 6,857 \\
\hline Preg. 3 & 5,996 & 1,778 & 0 & 9,735 & 9,524 & 7,407 & 8,274 \\
\hline Preg. 4 & 8,872 & 5,511 & 9,735 & 0 & 9,524 & 7,407 & 12,77 \\
\hline Preg. 5 & 18,182 & 8,571 & 9,524 & 7,407 & 0 & 5,714 & 11,87 \\
\hline Preg. 6 & 24,259 & 6,667 & 7,407 & 7,407 & 5,714 & 0 & 9,231 \\
\hline Preg. 7 & 16,233 & 6,857 & 8,274 & 12,77 & 11,87 & 9,231 & 0 \\
\hline
\end{tabular}

Fuente: Chipantiza (2017). 
En la tabla anterior, se muestra la relación que existe entre todas las preguntas utilizadas en el estudio de percepción de los miembros de la Asociación, ahí se observa que la mayoría de preguntas tienen un valor superior al valor teórico de chi cuadrado, por ello se concluye de acuerdo a los datos de la percepción de los asociados, que hay evidencia significativa que la gestión de calidad mejorará los procesos de producción de babaco.

\section{CONCLUSIONES}

De acuerdo al diagnóstico de los procesos de producción por medio de los diagramas de procesos de producción existen demoras, inspecciones, transporte, operaciones, innecesarias, largos tiempos de producción, mismos que son causados por la inexistencia de procedimientos que estandaricen las actividades a realizar.

Se identificó los lineamientos de calidad del producto valorado fundamentalmente por su uniformidad, madurez y ausencia de defectos, el color, la viscosidad y el rendimiento industrial como materia prima del producto terminado (babaco), en la Asociación. de productores Autónomos Rio Chico el Rosal a través del cual cada productor desarrolle

Se diseñó el proceso de producción del babaco en el cual se identifica desde su etapa inicial que es la preparación del suelo, diseño del huerto, plantación, labores culturales y pre culturales, cosecha postcosecha y distribución con el fin de asegurar la calidad del producto con la implementación de un sistema de gestión de calidad basado en la norma ISO para mejorar los procesos de producción de la Asociación de productores Autónomos Rio Chico el Rosal. 


\section{REFERENCIAS CONSULTADA}

Auquillas, A. (2016). Proyecto de mantenimiento y mejoras del sistema de gestión de la calidad de la empresa Flexiplast S.A bajo la Norma ISO 9001.Universidad Central del Ecuador. https://n9.cl/bbhs8

Camisón, C., Cruz, S., \& González, T. (2014). Gestión de la Calidad- conceptos, enfoques, modelos y sistemas. Editorial Pearson Prentice Hall. España

Casañas, J. (2015). La responsabilidad social y su incidencia en la producción de pantalones en la empresa Ram Jean's de la ciudad de Pelileo. Ambato. Editorial Universidad Técnica de Ambato. Ecuador.

Cuatrecasas, L. (2012). Organización de la producción y dirección de operaciones. Editorial Díaz de santos. España

González, S. (2011). Sistemas integrados de gestión, un reto para las pequeñas y medianas empresas. Revista Redalyc. Vol: 9, NUm.1, 69-89.

Guaipatin, C., \& Schwartz, L. (2014). Ecuador- Análisis del Sistema Nacional de Innovación. Impreso en Washington D.C: BID.

Gutiérrez, H. (2014). Calidad Total y Productividad. Cuarta edición. Editorial McGrawHill/Interamericana editores, S.A. de C.V. México.

Hernández, R., Fernández, C., \& Baptista, P. (2013) Metodología de la Investigación. Sexta edicion. Editorial. McGraw- HILL Interamericana. Mexico.

Instituto Nacional de Investigaciones Agropecuarias. (2012). INIAP. Obtenido de http://www.iniap.gob.ec/web/fruticultura/

ISO 2015. (2015). Sistema de Gestión de Calidad- Fundamentos y Vocabulario. Ginebra: ISO 2015. Cuarta edición.

ISO 9000; 2005. (2005). umc. Obtenido de https://n9.cl/wsleg

Mateo, C. (2010). Gestionpolis. Obtenido de https://n9.cl/itx3g

Montilla, O., \& Herrera, L. (2006). El deber ser de la Auditoría. Revista Scielo, Vol:22, Num. 98. Cali. Colombia.

Pérez, M. (2014). Estandarización de Procesos de la Empresa Textiles Técnicos. Ambato. Editorial Universidad Técnica de Ambato.Ecuador. 


\section{CIENCIAMATRIA}

Revista Interdisciplinaria de Humanidades, Educación, Ciencia y Tecnología Año VII. Vol. VII. N¹. Edición Especial. 2021 Hecho el depósito de ley: pp201602FA4721

ISSN-L: 2542-3029; ISSN: 2610-802X

Universidad Nacional Experimental Francisco de Miranda (UNEFM). Santa Ana de Coro. Venezuela

Juan Gabriel Chipantiza-Masabanda; Amanda Elizabeth Bonilla-Bonilla; Byron Stalin Rojas-Oviedo

(C2021 por los autores. Este artículo es de acceso abierto y distribuido según los términos y condiciones de la licencia Creative Commons Atribución-NoComercial-Compartirlgual 4.0 Internacional (CC BY-NC-SA 4.0)

(https://creativecommons.org/licenses/by-nc-sa/4.0/) 